\title{
EFECTO DE LABRANZA Y MANEJO DE MATERIALES ORGÁNICOS SOBRE ALGUNAS PROPIEDADES FÍSICAS Y QUÍMICAS DE UN VERTIC ENDOAQUEPT DEL VALLE DEL SINÚ, CÓRDOBA COLOMBIA
}

\section{EFFECT OF TILLAGE AND MANAGEMENT OF ORGANIC MATERIALS ON SOME PHYSICAL AND CHEMICAL PROPERTIES OF A VERTIC ENDOAQUEPT AT SINU VALLEY, CORDOBA COLOMBIA}

\author{
Bernardo Prieto ${ }^{1}$, José A. Peroza ${ }^{2}$, Garnierd Grandet ${ }^{3}$ \\ Recibido para evaluación: Noviembre 26 de 2009 - Aceptado para publicación: Noviembre 4 de 2010
}

\begin{abstract}
RESUMEN
Se evaluó el efecto de tres sistemas de labranza y tres aportes de materia orgánica sobre algunas propiedades físicas y químicas de un Vertic Endoaquept y sobre el rendimiento de los cultivos de maíz (Zea mays L.) y algodón (Gossypium hirsutum L.). Los sistemas de labranza fueron: convencional, vertical con cincel y siembra directa. Se utilizaron como fuentes de materia orgánica (MO) los residuos de cosecha (RC); RC + pollinaza y RC + bovinaza. Se analizaron las características físicas: densidad aparente (Da), porosidad Total (PT), resistencia mecánica a penetración de raíces (RMP) y conductividad hidráulica saturada (Ks) y químicas de: contenidos de $\mathrm{MO}$, fósforo $(\mathrm{P})$ y potasio $(\mathrm{K})$. Los resultados indican que los tratamientos de labranza no produjeron cambios significativos $(\mathrm{P} \geq 0,05)$ sobre las propiedades físicas $\mathrm{Da}, \mathrm{PT}, \mathrm{RMP}$ y Ks. La MO se incrementó de $2,24 \%$ a 2,67\%, 2,89\% y 2,98\% cuando se aplicaron los restos de cosecha, pollinaza y bobinaza, respectivamente. Igualmente, el P pasó de 25,4 ppm a 173,1, 229,9 y 202,8 ppm. El rendimiento de los cultivos registró diferencias significativas $(P \leq 0,05)$ a partir del segundo año de iniciadas las aplicaciones. En maíz, las aplicaciones de pollinazay bobinaza registraron rendimientos de 7450 y $7363 \mathrm{~kg} \mathrm{ha}^{-1}$ comparados con $6904 \mathrm{~kg} \mathrm{ha}^{-1}$ donde solo se aplicaron los restos de cosecha. En algodón, los mayores rendimientos se lograron con aplicación de pollinaza y bobinaza con 3255 y $3131 \mathrm{~kg} \mathrm{ha}^{-1}$, respectivamente. La incorporación de materiales orgánicos mejora la oferta edáfica y los rendimientos de los cultivos estudiados.
\end{abstract}

Palabras clave: Sistemas de labranza, restos de cosecha, pollinaza, bobinaza, algodón, maíz.

\footnotetext{
IIngeniero Agrónomo Ph.D. Profesor titular, Departamento de Ingeniería Agronómica y Desarrollo Rural, Universidad de Córdoba, Montería, Córdoba - Colombia. E_mail: bprieto@correo.unicordoba.edu.co

${ }^{2}$ Ingeniero Agrónomo y Agrícola, Profesor titular, Departamento de Ingeniería Agronómica y Desarrollo Rural, Universidad de Córdoba, Montería, Córdoba - Colombia

${ }^{3}$ Ingeniero Agrónomo MSc. Profesor catedrático, Departamento de Ingeniería Agronómica y Desarrollo Rural, Universidad de Córdoba, Montería, Córdoba - Colombia
} 


\begin{abstract}
The effect of three tillage systems and three sources of organic matter on some physical and chemical properties of a Vertic Endoaquept soil, and yield corn (Zea mays L) and cotton (Gosypium hirsutum L) was evaluated. The tillage systems were: conventional, vertical till with chisel plow, and direct sowing. The sources of organic matter $(\mathrm{MO})$ were crop residues $(\mathrm{RC}), \mathrm{RC}+$ poultry litter and $\mathrm{RC}+$ plus manure. The physical properties evaluated were: bulk density (Da), total porosity (PT), mechanical resistance to penetration of roots (RMP), saturated hydraulic conductivity (Ks). Chemical properties such organic matter contents $(\mathrm{MO})$, phosphorus (P) and potassium (K). After two years of management, the results showed that changes produced by tillage system on the physical properties (Da, PT, RMP and Ks) were temporary, because at the end of the crops cycle significant difference were not registrated ( $P \geq 0,05)$. The $M O$ increased from 2,24\% to 2,67, 2,89 and 2,98\% when were applied crops residues, poultry litter and manure respectively. In the same order the $\mathrm{P}$ increased from 25,4 ppm to $173,1,229,9$ and $202,9 \mathrm{ppm}$. Significant differences $(\mathrm{P} \leq 0,05)$ were registered in yield cotton and corn crops in the second year. In Corn, supply of poultry litter and manure yield 7450 and 7363 $\mathrm{kg} \mathrm{ha}^{-1}$ compared to $6904 \mathrm{~kg} \mathrm{ha}^{-1}$ with only crop residues. In cotton, the highest yields were observed with applications of poultry litter and manure with 3255 and $3131 \mathrm{~kg} \mathrm{ha}^{-1}$ respectively. Addition of OM increased yield and the levels of OM in the soil.
\end{abstract}

Key words: Tillage Systems, Crops Residues, poultry Litter, Manure, Cotton, Corn.

\section{INTRODUCCIÓN}

En la región del Valle medio del río Sinú la actividad agrícola principal la constituyen los cultivos de maíz (primer semestre) y algodón (segundo semestre) con una extensión aproximada de 25.0000 ha. La intensidad del laboreo y la forma indiscriminada como se ha venido practicando a lo largo de más de 30 años, ha conducido a la degradación estructural de los suelos con reducción de los contenidos de materia orgánica a niveles bajos y muy bajos y el consiguiente deterioro de las propiedades físicas como la densidad aparente, porosidad, infiltración, conductividad hidráulica, retención de humedad, alta resistencia a la penetración de raíces, aumento en las cantidades de fertilizantes utilizados y por consiguiente aumentos en los costos de producción (Grandet 2001).

Muchos trabajos sobre sistemas de labranza se han llevado a cabo en diferentes partes del mundo, con resultados diversos. Sin embargo, en la costa Caribe Colombiana se reportan pocos trabajos de investigación en sistemas de labranza, en la revisión realizada se encontraron los trabajos de García (1991); Bonilla y Murillo (1998); Bonilla y Vanegas (1998); García y Duran (1998) realizados en la región algodonera del Cesar; Martínez et al. (2003) en la región del Sinú. Aunque la información recolectada es valiosa, en su mayoría corresponde a experimentos con un año de manejo.

Es necesario adelantar trabajos de investigación que a mediano plazo, permitan ofrecer a los agricultores y asistentes técnicos alternativas de producción competitiva en costos, sostenibles en lo ecológico y que sean el resultado de investigaciones locales. De continuar con el manejo actual, cada año será necesario ir incrementando las cantidades de fertilizantes para mantener altas producciones, pudiendo llegar a niveles de fertilización no rentables, 
sobretodo si se tiene en cuenta el aumento en el precio de éstos. Aunque las técnicas de la siembra directa y la labranza de conservación se han venido aplicando con éxito en otras regiones, el proceso de cambio de labranza convencional a siembra directa involucra investigación permanente "in situ", pues los efectos del laboreo sobre las propiedades del suelo varían en un amplio rango según las condiciones climáticas (Heard et al. 1988). Por lo tanto, si se desea obtener rendimientos rentables, de buena calidad y con una producción sostenible y con capacidad para competir en los mercados internos y externos, es necesario, entre otros factores, que el suelo sea capaz de suministrar a la planta el agua y los nutrientes esenciales en cantidades adecuadas y oportunas. Para ello debe poseer buenas propiedades físicas y químicas (Lora 1998).

Teniendo en cuenta lo anterior, el presente estudio tuvo como objetivo analizar a mediano plazo la evolución de las propiedades físicas, y químicas de un suelo del Valle del Sinú y su influencia sobre el rendimiento de los cultivos de maíz y algodón, por efecto del laboreo, el manejo de los residuos de cosecha y la adición de materia orgánica.

\section{MATERIALES Y MÉTODOS}

\section{Localización}

El presente trabajo de investigación se realizó en la Cooperativa Agropecuaria de Córdoba COAGROCOR ubicada en el municipio de Cereté, Departamento de Córdoba Colombia. Coordenadas geográficas $8^{\circ}$ 53' 05" Latitud Norte y $75^{\circ} 47^{\prime} 17^{\prime \prime}$ Longitud Oeste. 16 m de altura sobre el nivel del mar. Zona agro climáticas Bosque Seco Tropical (BsT), según el modelo Holdridge, con una precipitación promedio de $1700 \mathrm{~mm}$ anuales distribuidos en los meses de abril a noviembre y periodo seco de diciembre a marzo, la temperatura media anual es de $28^{\circ} \mathrm{C}$. Suelo de textura franco arcillosa, clasificado como un Vertic Endoaquept (USDA 1999), con más de 30 años de cultivo intensivo para lograr cada año una cosecha de maíz y una de algodón. El sistema de labranza utilizado ha sido el laboreo convencional. El estudio tuvo una duración de dos ciclos de cultivo (dos años agrícolas).

\section{Tratamientos}

Los tratamientos de labranza y materia orgánica aplicados fueron:

$\mathrm{T} 1$ = Labranza Convencional $(\mathrm{LC})+$ Residuos de Cosecha (RC).

$\mathrm{T} 2$ = Labranza Convencional $(\mathrm{LC})+$ Residuos de Cosecha $(\mathrm{RC})$ + Pollinaza

$\mathrm{T} 3$ = Labranza Convencional $(\mathrm{LC})+$ Residuos de Cosecha $(R C)+$ Bobinaza

$\mathrm{T} 4$ = Labranza Vertical (LV) + Residuos de Cosecha (RC).

T5 = Labranza Vertical (LV) + Residuos de Cosecha $(\mathrm{RC})$ + Pollinaza

T6 = Labranza Vertical (LV) + Residuos de Cosecha $(\mathrm{RC})+$ Bobinaza

$\mathrm{T} 7=$ Siembra Directa $(\mathrm{SD})+$ Residuos de Cosecha (RC).

$\mathrm{T} 8=$ Siembra Directa $(\mathrm{SD})+$ Residuos de Cosecha $(\mathrm{RC})+$ Pollinaza.

T9 = Siembra Directa $(\mathrm{SD})+$ Residuos de Cosecha $(\mathrm{RC})+$ Bobinaza.

La labranza convencional (LC) consistió en dos pases de rastra pesada, un pase de rastrillo y la siembra se realizó con una sembradora convencional. 
La labranza vertical (LV) consistió en un pase de cincel rígido y un pase de cincel vibratorio sobre los residuos de la cosecha anterior. La siembra se realizó con sembradora neumática de siembra directa.

La siembra directa (SD) se realizó con una sembradora neumática diseñada para esta labor, sobre los residuos de la cosecha cortados previamente con una desbrozadora.

Los tratamientos de materia orgánica $(\mathrm{MO})$ fueron aplicados un mes antes de la preparación del terreno, se utilizaron dos abonos orgánicos, pollinaza y bobinaza como fuentes externas, a razón de $4.000 \mathrm{~kg} \mathrm{ha}^{-1}$ de materia seca y los residuos de la cosecha que fueron incorporados al suelo. Tanto los tratamientos de labranza como la materia orgánica se aplicaron solamente en el primer semestre, antes de la siembra del cultivo de maíz.

Los cultivos plantados fueron maíz en el primer semestre y algodón en el segundo semestre, por ser éstos los dos cultivos comerciales y tradicionales en la región.

Los tratamientos se establecieron sobre un arreglo en parcelas divididas con tres repeticiones. El área de las parcelas principales fue de $832 \mathrm{~m}^{2}$ y sobre ellas se establecieron los tratamientos de laboreo. Cada parcela fue dividida en tres sub-parcelas con un área de 278 m2, en éstas se aplicó la materia orgánica. El área total del experimento ocupó 9360 m².

\section{Procedimientos}

\section{Caracterización del suelo del sitio del} experimento. Antes de establecer los tratamientos se realizó una caracterización inicial del suelo, para lo cual se construyó una calicata de 1 $\mathrm{m} \times 1 \mathrm{~m}$ hasta la profundidad de 0,7 m. Se evaluaron las propiedades físicas y se tomaron las muestras necesarias para la determinación de las propiedades químicas en el laboratorio.

Después de dos años, en los cuales se aplicaron los tratamientos al suelo y se realizaron dos ciclos de cultivo maíz - algodón, se evaluaron nuevamente las variables en estudio.

Propiedades físicas. Las propiedades físicas evaluadas fueron densidad aparente (Da) por el método de los cilindros de volumen conocido (Coile 1936). Densidad real (Dr) por el método del picnómetro (IGAC 1990). La porosidad fue calculada a partir de la densidad aparente y la densidad real mediante la siguiente relación:

$$
\text { Porosidad }(\%)=\left(1-\frac{D a}{D r}\right) 100
$$

Resistencia mecánica a penetración medida con un penetrógrafo de cono marca Eijkelkamp con capacidad para registrar resistencias hasta de 5 MPa y hasta profundidad de $8 \mathrm{~m}$, el área de base del cono fue de $1,0 \mathrm{~cm}^{2}$.

Las tasas de infiltración y conductividad hidráulica fueron determinadas con infiltrómetros de tensión de disco (Perroux y White 1988).

Propiedades químicas. Para evaluar el efecto de los tratamientos sobre las propiedades químicas, al final del segundo ciclo de cultivos, se tomaron muestras de suelo de todas las unidades experimentales $y$ se Ilevaron al laboratorio de suelos de la Universidad de Córdoba donde se procesaron según la metodología del IGAC (1990) para las siguientes determinaciones: $\mathrm{pH}$, contenidos de 
materia orgánica, nitrógeno, fósforo, potasio, calcio, magnesio, azufre, sodio y elementos menores (hierro, zinc y manganeso).

Caracterización de la Biomasa. En cada ciclo, que correspondió a un cultivo de maíz y uno de algodón, el aporte de $\mathrm{MO}$ al suelo provino de los residuos (socas) de los cultivos de maíz y algodón más el aporte de las malezas que logran crecer entre la finalización de un cultivo y el inicio del siguiente; además, el aporte de los abonos pollinaza y bobinaza, en los tratamientos donde se aplicaron. La cuantificación de estos materiales arrojó resultados importantes, así por ejemplo, se pudo establecer que la soca de un cultivo de maíz genera en promedio $12.300 \mathrm{~kg} \mathrm{ha}^{-1}$ de materia seca, mientras que la del algodón produjo en promedio $6.600 \mathrm{~kg}$ ha-1 y que las malezas pueden llegar a dar un aporte de $4.300 \mathrm{~kg}$ ha-1 o sea que en un ciclo de cultivos puede lograrse un significativo aporte de 23.200 kg ha-1, que de no ser quemado genera una actividad importante en el suelo.

Rendimiento de los cultivos. La respuesta de los cultivos a los tratamientos se evaluó a través de un muestreo de rendimiento, cosechando los dos surcos centrales de cada unidad experimental y en cada cultivo. Esta variable fue medida en los dos ciclos de cultivo maízalgodón (dos años agrícolas) y se expresó en kg $\mathrm{ha}^{-1}$, con base en un $15 \%$ de humedad para el maíz y $10 \%$ para el algodón.

Análisis estadístico. Se utilizó el programa SAS. A cada variable se le realizó la prueba de normalidad y homogeneidad de varianza, un análisis de varianza individual y su correspondiente prueba de medias de Duncan.

\section{RESULTADOS Y DISCUSIÓN}

La determinación de las propiedades físicas al iniciar el experimento acuso los siguientes valores: para la densidad aparente $1.58 \mathrm{Mg}$ $\mathrm{m}^{-3}$, la resistencia a penetración de raíces 1,60 MPa, porosidad total 38,2\%, conductividad hidráulica $1,76 \mathrm{~cm} \mathrm{~h}^{-1}$. De igual manera, los análisis de las características químicas proporcionaron los siguientes valores: contenido de MO 2,24\%, fósforo 25,4 ppm, potasio 0,99 meq $100 \mathrm{~g}^{-1}$ y calcio $15 \mathrm{meq}$ $100 \mathrm{~g}^{-1}$ de suelo.

Al efectuar el análisis de varianza, la interacción de los sistemas de labranza y las fuentes de materia orgánica no mostró diferencias significativas ( $P \geq 0,05)$, razón por la cual los resultados de la labranza se presentan separadamente de los resultados de las fuentes de materia orgánica.

Efectos sobre algunas propiedades físicas del suelo. El análisis de varianza para la densidad aparente (Da), porosidad total (PT), resistencia mecánica a penetración de raíces (RMP) y conductividad hidráulica saturada (Ks) a la profundidad de 0-20 cm en los diferentes sistemas de labranza utilizados, mostró ausencia de diferencias significativas $(P \geq 0,05)$ (Tabla 1); resultados que concuerdan con los reportados por Amezquita et al. (1998) quienes no encontraron diferencias significativas en los valores de la densidad aparente entre los diferentes sistemas de labranza en un vertisol del Valle del Cauca. Igual situación, reportan Martínez et al. (2003) en un estudio en suelos del Valle de Sinú. La mejora en las propiedades físicas del suelo es un proceso lento y en el caso de este estudio, muy probablemente, dos años 
TEMAS AGRARIOS - Vol. 15:(2) Julio - Diciembre 2010 (27 - 36)

Tabla 1. Efecto de tres sistemas de labranza sobre algunas propiedades físicas a la profundidad de 0- $20 \mathrm{~cm}$ en un suelo del Sinú Medio 2006-2008

\begin{tabular}{lcccc}
\hline $\begin{array}{c}\text { Sistema de } \\
\text { Labranza }\end{array}$ & $\begin{array}{c}\text { Densidad aparente } \\
(\text { Mg m-3) }\end{array}$ & $\begin{array}{c}\text { Porosidad Total } \\
(\%)\end{array}$ & $\begin{array}{c}\text { Resistencia a } \\
\text { penetración (Mpa) }\end{array}$ & $\begin{array}{c}\text { Humedad } \\
(\%)\end{array}$ \\
\hline SD & $1,40 \mathrm{a}$ & $44,61 \mathrm{a}$ & $1,58 \mathrm{a}$ & $29,45 \mathrm{a}$ \\
LV & $1,35 \mathrm{a}$ & $45,41 \mathrm{a}$ & $1,37 \mathrm{a}$ & $32,01 \mathrm{a}$ \\
LC & $1,36 \mathrm{a}$ & $47,60 \mathrm{a}$ & $1,47 \mathrm{a}$ & $30,09 \mathrm{a}$ \\
C.V. $\%$ & 3,29 & 3,9 & 14,47 & 5,92 \\
\hline
\end{tabular}

SD = Siembra Directa, LV = Labranza Vertical, LC = Labranza Convencional. CV = Coef. de Variación. Valores seguidos de la misma letra en la misma columna son estadísticamente iguales $(P \geq 0,05)$

no son suficiente tiempo para lograr una mejora significativa, sin embargo, los resultados, por lo menos, muestran esa tendencia. Muchos estudios en suelos de textura fina muestran mayor aumento de la densidad bajo sistemas de no laboreo que con algún tipo de labranza (Roth et al., 1988; Lal et al., 1989). Sin embargo, en algunos otros estudios no se han encontrado diferencias (Blevins et al., 1983; Bauder y Black, 1981). Igual situación se presenta con las tasas de infiltración, en algunos estudios con tratamientos de no laboreo, éstas han sido mayores que en los de laboreo (Chan y Heenan, 1993; Dao 1993; Moreno et al. 1997), aunque en otros casos se ha encontrado lo contrario (Gómez et al. 1999; Kribaa et al. 2001), lo anterior demuestra que las propiedades físicas del suelo varían en un amplio rango.

Los restos de cosecha y las aplicaciones de pollinaza y bovinaza no contribuyeron al mejoramiento de las propiedades físicas durante el tiempo que duró el experimento, aunque si se observó una tendencia hacia el mejoramiento. En este orden de ideas, Reddy et al. (2001) resaltan que la pollinaza fresca o compostada es una fuente importante de $\mathrm{N}$ y mejora los contenidos de materia orgánica $y$ a largo plazo las propiedades físico-químicas del suelo.

\section{Efectos sobre algunas propiedades químicas}

del suelo. Los tratamientos de labranza no logaron introducir mejoras significativas en las características químicas de este suelo durante el tiempo que duró el experimento. Estos resultados están de acuerdo con los obtenidos por Motta et al. (2001) quienes no encontraron un claro efecto de los sistemas de laboreo sobre las propiedades químicas del suelo, aunque si reportaron aumentos de los contenidos de materia orgánica en los tratamientos de labranza de conservación con respecto a los de laboreo convencional.

El efecto del manejo de los restos de cosecha y la adición de pollinaza y bobinaza sobre las propiedades químicas del suelo analizadas al final del estudio, se presentan en la tabla 2, en ella se puede observar la ausencia de significancia entre los tres materiales. Sin embargo, cuando la comparación se hace entre el contenido de MO inicial 2,24\%, con los contenidos de $\mathrm{MO}$ alcanzados al final de los dos ciclos de cultivo, 2,67\% con el solo manejo de residuos de cosecha, 2,89\% agregando pollinaza y $2,98 \%$ agregando 
Prieto - Labranza sobre las propiedades del suelo

Tabla 2. Efecto de tres fuentes de materia orgánica sobre algunas propiedades Químicas a la profundidad de 0- 20 cm en un suelo del Sinú Medio 2006-2008

\begin{tabular}{lcccccc}
\hline \multirow{2}{*}{$\begin{array}{c}\text { Fuentes de } \\
\text { Materia Orgánica }\end{array}$} & Materia Orgánica & $\mathrm{P}$ & $\mathrm{K}$ & $\mathrm{Ca}$ & $\mathrm{Mg}$ \\
\cline { 2 - 7 } & $\%$ & $\mathrm{ppm}$ & \multicolumn{3}{c}{ Meq 100g-1 de suelo } \\
\hline Contenidos iniciales & $2,24 \mathrm{~b}$ & $25,4 \mathrm{~b}$ & $0,99 \mathrm{~b}$ & $15,0 \mathrm{~b}$ & $9,0 \mathrm{a}$ \\
Restos de cosecha (RC) & $2,67 \mathrm{a}$ & $173,18 \mathrm{a}$ & $2,44 \mathrm{a}$ & $16,33 \mathrm{a}$ & $7,94 \mathrm{ab}$ \\
RC + Pollinaza & $2,89 \mathrm{a}$ & $229,94 \mathrm{a}$ & $2,19 \mathrm{a}$ & $17,05 \mathrm{a}$ & $7,44 \mathrm{~b}$ \\
RC + Bobinaza & $2,98 \mathrm{a}$ & $202,83 \mathrm{a}$ & $2,82 \mathrm{a}$ & $16,11 \mathrm{ab}$ & $7,72 \mathrm{ab}$ \\
CV \% & 11,31 & 59,02 & 27,36 & 6,24 & 15,14 \\
\hline
\end{tabular}

Valores seguidos de la misma letra en la misma columna son estadísticamente iguales $\mathrm{P} \geq 0,05$ )

bobinaza, los resultados muestran que los tres tratamientos fueron estadísticamente diferentes con la situación inicial.

En concordancia con el aumento en los contenidos deMO, seregistrótambién unaumentosignificativo en el $\mathrm{P}$ disponible alcanzando niveles de 229,9 ppm y 202,8 ppm en los tratamientos donde se aplicó pollinaza y bobinaza respectivamente, pero también los restos de cosecha lograron aumentos de 173,2 ppm.

Las bases intercambiables presentaron valores altos: el $\mathrm{K}$ aumentó en contenido debido principalmente a sus pocos procesos de pérdida, no se aprecia una tendencia que relacione a las fuentes de materia orgánica debido a que el $\mathrm{K}$ del suelo es de origen mineral y su forma intercambiable es consecuencia del intemperismo que se acelera con las aplicaciones de materia orgánica, reflejándose en los mayores contenidos de K intercambiable. El Ca aumentó con relación a los contenidos iniciales. El Mg bajo significativamente cuando se aplicó la pollinaza, aunque, sigue presentando valores altos pero conservando una relación $\mathrm{Ca} / \mathrm{Mg}$ adecuada para los cultivos. Los resultados de esta investigación muestran que la aplicación de materiales orgánicos y el manejo de los restos de las cosechas en los suelos del Valle Medio del Sinú, mejora la oferta edáfica para el desarrollo y producción de los cultivos de maíz y algodón y confirman lo reportado por muchos investigadores como Green y Blackmer (1995), Bayer et al. (2001), Carrie et al. (2003) quienes han sostenido que los materiales orgánicos incorporados al suelo mejoran los procesos químicos e incrementan la disponibilidad de nutrientes.

\section{Efectos sobre el rendimiento de los cultivos}

Los rendimientos de los dos ciclos de cultivos, maíz-algodón, durante los años 2006/07 y 2007/08 se resumen en la tabla 3.

Los rendimientos de los cultivos de maíz algodón no se afectaron significativamente $(P \geq 0.05)$ por los tres sistemas de laboreo en ninguno de los ciclos realizados. Las fuentes de materia orgánica tampoco mostraron diferencias significativas en los rendimientos del primer ciclo de cultivos. Pero para la cosecha de maíz 2007 y algodón 2007/08 si se observaron diferencias significativas (P 0.05), en los tratamientos donde se aplico materia orgánica. Se observó que donde se aplicó 
TEMAS AGRARIOS - Vol. 15:(2) Julio - Diciembre 2010 (27 - 36)

Tabla 3. Efecto de tres fuentes de Materia Orgánica sobre los rendimientos Promedios de los cultivos de Maíz y algodón en el Sinú Medio 2006-2008

\begin{tabular}{lcccc}
\hline \multirow{2}{*}{$\begin{array}{c}\text { Fuentes de } \\
\text { Materia Orgánica }\end{array}$} & Maíz & 2007 & $2006 / 07$ & $2007 / 08$ \\
\cline { 2 - 5 } & 2006 & $6904 \mathrm{~b}$ & $2767 \mathrm{a}$ & $2707 \mathrm{~b}$ \\
\hline Restos de cosecha & $7419 \mathrm{a}$ & $7450 \mathrm{a}$ & $2997 \mathrm{a}$ & $3255 \mathrm{a}$ \\
$\mathrm{RC}+$ Pollinaza & $7628 \mathrm{a}$ & $7363 \mathrm{a}$ & $3112 \mathrm{a}$ & $3131 \mathrm{a}$ \\
RC + Bobinaza & $7552 \mathrm{a}$ & 8,74 & 9,28 & 11,35 \\
CV \% & 6,82 &
\end{tabular}

Valores con la misma letra en la misma columna son estadísticamente iguales $(\mathrm{P} \geq 0,05)$

pollinaza y bobinaza, los rendimientos del maíz, en la cosecha 2007, fueron de 7450 y $7363 \mathrm{~kg} \mathrm{ha}^{-1}$ respectivamente, frente a $(6904 \mathrm{k}$ ha $^{-1}$ ) donde sólo se consideraron los residuos de cosecha. Igual situación, se presentó en el cultivo de algodón, 2007/8, los mayores rendimientos se alcanzaron con aplicación de pollinaza y bobinaza (3255 y $3131 \mathrm{~kg} \mathrm{ha}^{-1}$ ) respectivamente, contra $2707 \mathrm{~kg} \mathrm{ha}^{-1}$ donde no se aplicó. Esto guarda relación con la mejora en las propiedades, químicas del suelo en el tiempo y confirma lo encontrado por Reddy et al (2001).

\section{CONCLUSIONES}

Para el suelo y las condiciones en que se realizó el experimento se pueden deducir las siguientes conclusiones:

Los tres sistemas de labranza estudiados no registraron diferencias significativas sobre las propiedades físicas, químicas ni sobre los rendimientos de los cultivos de maíz y algodón y se muestra que dos años no son suficientes en este suelo, para lograr mejoras significativas.

El manejo de los residuos de cosecha y las aplicaciones de pollinaza y bobinaza aumentaron los contenidos de materia orgánica oxidable, de fósforo, de potasio y de calcio, mantuvieron una relación $\mathrm{Ca} / \mathrm{Mg}$ aceptable y no presentaron problemas de acidez ni basicidad en el suelo. En las condiciones del Valle Medio del Sinú, las socas de maíz, algodón y las malezas regresan al suelos una cantidad de biomasa estimada en 23.000 kg ha $^{-1}$ de materia seca que ofrece un sustrato permanente para la biota del suelo, pues su calidad y tipo indica una descomposición gradual.

Las aplicaciones de pollinaza y bobinaza aumentaron los rendimientos de los cultivos de maíz y algodón a partir del segundo año de iniciadas las aplicaciones.

Teniendo en cuenta el reciclaje de nutrientes proveniente de las malezas, residuos de cultivos y las adiciones de abonos orgánicos es posible disminuir el uso de fertilizantes inorgánicos, principalmente el fósforo y potasio.

\section{REFERENCIAS}

Amézquita, E. 1998. Propiedades físicas de los suelos de los Ilanos orientales y sus requerimientos de labranza. Memorias 
I. Encuentro Nacional de Labranza de Conservación. Editora Guadalupe Ltda, Villavicencio, p145-174.

Bauder, A., Black, A.L. 1981. Soil carbon , nitrogen, and bulk density comparison in two cropland tillage systems after 25 years and in virgin grassland. SoilSci. Soc. Am. J. 45: 1166-1170.

Bayer C, Martin-Neto L, Mielniczuk J, Pillon CN, Sangoi L. 2001. Changes in soil organic matter fractions under subtropical no-till cropping systems.Soil. Sci. Soc. Am. J. 65: 1473-1478.

Blevins, R.L., Thomas, G.W., Smith, M.S., Frye, W.W., Cornelius, P.L. 1983. Changes in soil properties after 10 years continuous no-tilled and conventionally tilled corn. SoilTill. Res. 3: 135-146.

Bonilla, R. y Murillo, J. 1998. Desarrollo de sistemas de manejo para la recuperación de suelos compactados de los departamentos de la Guajira, Cesar y Magdalena. Memorias I. Encuentro Nacional de Labranza de Conservación. Editora Guadalupe Ltda, Villavicencio, p195-204.

Bonilla, R. y Vanegas, N. 1998. Efecto de sistemas de labranza sobre las propiedades físicas y producción del algodonero en suelos compactados del valle del Cesar. Memorias I. Encuentro Nacional de Labranza de Conservación. Editora Guadalupe Ltda, Villavicencio, p205-206.
Chan, K.Y., Heenan, D.P. 1993. Surface hydraulic properties of a Red Earth under continuous cropping with different management practices.Aust. J. Soil Res. 31: 13-24

Carrie A, Laboski M, Lamb JA. 2003. Changes in soil test phosphorus concentration after application of manure or fertilizer. Soil Sci. Soc. Am. J. 67: 544-554.

Coile, T.S. 1936. Soil samplers.Soil Sci.42: 139-141.

Dao, Thant H., 1993. Tillage and winter wheat residue management effects on water infiltration and storage.SoilSci. Soc. Am. J. 57: 1586-1595.

García, J. y Durán, R. 1998. Evaluación de varios sistemas de labranza en un suelo algodonero del valle del Cesar. Memorias I. Encuentro Nacional de Labranza de Conservación. Editora Guadalupe Ltda, Villavicencio, p241-257.

García, J. 1991. Efectos del laboreo en algunas propiedades físicas de los suelos del valle medio del Sinú. RevistaSuelosEcuatoriales 21 (1): 51-54.

Gómez, J.A., Giráldez, J.V., Pastor, M., Fereres, E. 1999. Effects of tillage method on soil physical properties, infiltration and yield in an olive orchard.SoilTill. Res. 52:167-175.

Grandet, G. 2001. Evaluación de las propiedades físicas de los suelos de varias fincas agrícolas del Sinú medio. Mineografiado, p46. 
Green CJ, Blackmer AM. 1995. Residue descomposition effect on nitrogen availability to corn following corn or soybean. Soil Sci Soc. Am. J. 59: 1056-1070

Heard, J.R., Kladivko, E.J., Mannering, J.V. 1988. Soil macroporosity, hydraulic conductivity and air permeability of silty soil under long-term conservation tillage in Indiana.SoilTill. Res. 11: 1-18.

IGAC. 1990. Métodos analíticos del Laboratorio de Suelos. Quinta edición. IGAC. Bogotá, p 502.

Kribaa, M., Hallaire, V., Curmi, P., Lahmar, R. 2001. Effect of various cultivation methods on the structure and hydraulic properties of a soil in a semi-arid climate. Soil Till. Res. 60, 43-53.

Lal, R., Logan, T.J., Fausey, N.R., Eckert. D.J. 1989. Long-term tillage and well traffic effects on a poorly drained MollicOchraqualf in northwest Ohio. SoilTill. Res.14, 341-358.

Lora, R.1998. Propiedades físicas y químicas del suelo.Memorias I. Encuentro Nacional de Labranza de Conservación. Editora Guadalupe Ltda, Villavicencio, p15-38.

Martínez, J.; Sánchez, C.; Roveda, G.; y Arrieta, A. 2003. Efecto de los sistemas de labranza sobre algunas propiedades hidrofísicas en un Endoaquept del sistema de producción maíz-algodón en el valle medio del Sinú. Memorias II. Encuentro Nacional de Labranza de Conservación, Villavicencio, p143-157.
Moreno, F., Pelegrin, F., Fernández, J.E. Murillo, J.M. 1997. Soil physics properties, water depletion and crops developments under traditional and conservation tillage in southern Spain. Soil Till. Res. 41, 25-42.

Motta, A. C. V., Reeves, D. W., Feng, Y., Burmester, C., and Raper, R.L. 2001. Management systems to improve soil quality for cotton production in a degraded silt loam soil in Alabama (USA) In: I World Congress on Conservation Agriculture. p219-222.

Perroux, K.M., White, I. 1988. Designs for disc permeameters. Soil Sci. Soc. Am. J. 52, 1205-1215.

Reddy, K.C., Nyakatawa, E.Z., and Malik, R.K. 2001. Strategies to Sustain Cotton Production Systems in SE USA.In: I World Congress on Conservation Agriculture. p533-537.

Roth, C.H., Meyer, B., Frede, G.J., Derpsch, R. 1988. Effect of mulch rates and tillage systems on infiltrability and other soil physical properties of an Oxisol in Parana, Brasil.Soil Till. Res. 11, 81-91.

USDA. 1999. Soil Taxonomy, Second Edition. Natural Resources Conservation Service. ftp://ftp-fc.sc.egov.usda.gov/NSSC/Soil_ Taxonomy/tax.pdf [12 Julio de 2010]. 